\title{
Resistive Index of Intrarenal Artery in Evaluation of Diabetic Nephropathy
}

\author{
Shirin $\mathrm{M}^{1}$, Sharif $\mathrm{MM}^{2}$, Gurung $\mathrm{A}^{3}$, Datta $\mathrm{A}^{3}$ \\ 1Department of Radiology and Imaging, Bangabandhu Sheikh Mujib Medical University, Shahbag, Dhaka, \\ 2BIRDEM, Shahbag, Dhaka, 3Bangabandhu Sheikh Mujib Medical University, Shahbag, Dhaka. \\ Email: m.shirin1970@gmail.com
}

\begin{abstract}
Diabetes mellitus is one of the systemic diseases affecting the kidneys. Diabetic nephropathy is a serious microvascular complication of diabetes mellitus. It is the most important cause of death in type I diabetic patients, of whom 30\%-40\% eventually develop end-stage renal failure and $40 \%$ of type II diabetics are at risk of developing diabetic nephropathy. So, diagnosis of diabetic nephropathy is paramount for the survivability of the diabetic patients not only because of the consequences of renal progression but also because of the strong association with the risk of developing cardiovascular disease. A total number of 53 subjects were enrolled in this present cross sectional study in the department of Radiology and Imaging, Bangabandhu Sheikh Mujib Medical University (BSMMU) in collaboration of Nephrology and Medicine of the same institution during two years (2011-13) aim to evaluate the diagnostic usefulness of renal resistive index (RI) by duplex Doppler ultrasonography for detection of renal dysfunction in diabetic patients. Clinically diagnosed diabetic patients having diabetic nephropathy referred to the department of Radiology and Imaging in BSMMU for ultrasonography of Kidneys, Ureters and Bladder (KUB) region or whole abdomen were selected as sample. Biochemical reports (Serum creatinine and Urinary albumin) and the RI value of intrarenal artery were correlated and analyzed. Only those patients biochemically were diagnosed as having diabetic nephropathy was included. Those with incomplete data, hydro nephrosis and renal calculus were excluded. Both the kidneys were visualized by commercially available real time scanner (GE Voluson) equipped with a curvilinear transducer operating at 3.5 MHz First Gray scale ultrasonography was done followed by Color Doppler of intra renal artery and then RI was measured. Majority (45.3\%) patients were in 6th decade with the mean age was of $52.66 \pm 7.4$ years and ranging from 38 to 65 years in patients. Male was found to be $54.7 \%$ of diabetic patients with male to female ratio 1.2:1. Resistive index of $(\geq 0.7)$ was found in $73.6 \%$ patients with diabetes with the mean resistive index of $0.71 \pm 0.04$. Positive correction between resistive index with serum creatinine $(r=0.581, \mathrm{p}<0.01)$ and albuminuria $(r=0.725, \mathrm{p}<0.01)$ were observed. It can be concluded that Resistive Index measured by duplex Doppler ultrasonography is useful diagnostic modality for detection of renal dysfunction in diabetic nephropathy patients. Resistive Index has value in identifying diabetic patients who are developing nephropathy and can be used as an additional diagnostic tool. Also it is well correlated with Serum Creatinine and Albuminuria which are the biochemical parameters to diagnose diabetic nephropathy.
\end{abstract}

Key words: Resistive Index, Duplex Color Doppler, Diabetic Nephropathy.

\section{Introduction}

Diabetic nephropathy is a serious microvascular complication of diabetes mellitus. It is estimated that the death due to renal disease is 17 times more common in diabetics than in non-diabetics. ${ }^{1}$ The hallmark of the renal lesion is a particular form of glomerulosclerosis, associated with arteriolar hyalinosis and interstitial fibrosis. These lesions are associated with clinical syndrome of proteinuria, hypertension and progressive renal failure. $^{2}$ Diabetic nephropathy is a clinical syndrome characterized by the Persistent albuminuria ( $>300 \mathrm{mg} / \mathrm{d}$ or $>200 \mu \mathrm{g} / \mathrm{min}$ ) that is confirmed on at least two occasions 3-6 months apart, Progressive decline in the glomerular 
filtration rate (GFR) and Elevated arterial blood pressure. ${ }^{3}$ Serum creatinine level more than $1.3 \mathrm{mg} / \mathrm{dl}$ was also considered as having diabetic nephropathy and patient having albumin in urine more than $300 \mathrm{mg} / \mathrm{dl}$ was considered to have microalbuminuria.

Conventionally diagnosis of diabetic nephropathy is made on clinical ground without a renal biopsy. Supportive clues are the normal or enlarged size of the kidneys, evidence of proliferative retinopathy and band urinary sediments. In addition, other biochemical examinations are also recommended such as urinary albumin, serum creatinine, creatinine clearance and urinary analysis for microhaematuria, leucocytes, casts. ${ }^{3,4}$

Resistive indices by duplex Doppler ultrasonography provides high quality and noninvasive display of importance in severe kidney diseases. It also predicts renal functional status in diabetic patient and also causing early detection of diabetic nephropathy. ${ }^{5}$ Thus it provides a numerical parameter for assessing renal functional status. Average RI value in intrarenal artery lie between $0.53-0.68$. If RI value is equal or above 0.70 , considers to be abnormal. ${ }^{6}$

Soldo et al. and Platt et al. found that resistive index correlate well with renal function, i.e. with serum creatinine level and creatinine clearance rates $^{5}$ and pathological values (i.e. $\geq 0.70$ ) were observed in $87 \%$ in the group with advanced nephropathy. ${ }^{6}$ Pathologic resistive indices may be detected in the earlier stages. An elevated RI (> or $=0.70)$ was associated with impaired renal function, increased proteinuria at 24 hours, and poor outcome. ${ }^{9-11}$

Kim et al. observed that there was statistically significant difference between resistive indices in diabetic patients with normal serum creatinine level and those in diabetic patients with elevated serum creatinine level $(\mathrm{P}<0.05)$ leading them to conclude that analysis of the Doppler spectrum of the intrarenal arteries in conjunction with careful evaluation of the renal cortical echogenicity might be helpful in sonographic differentiation of kidneys with preserved function and those with impaired function in patients with diabetes mellitus. ${ }^{7}$ Ohta et al. found that the RI and PI of the main renal arteries were significantly higher in patients with diabetic nephropathy. ${ }^{8}$
Histopathological changes affect mainly the vascular compartments in the kidneys of diabetic patients. ${ }^{12}$ Intrarenal Resistive Index is a measure of hemodynamic changes in the renal arteries. Alteration in the vascular compartments of the kidneys result in elevation of renal vascular resistance, manifested by increased values of the Doppler sonographic renal resistive indexes of intrarenal arteries. ${ }^{13}$ Pathological resistive indices may be detected in earlier stages. Resistive indices correlate with serum creatinine level and creatinine clearance rate. Renal RI correlated highly with serum creatinine concentration and creatinine clearance rate. ${ }^{5}$ An elevated RI ( $>$ or $=$ 0.70 ) was associated with impaired renal function, increased proteinuria at 24 hours and poor outcome. Sari et al. concluded that because the intrarenal RI shows a high level of correlation with serum creatinine concentration it can be used as a predictor in patient with advanced clinical diabetic nephropathy. ${ }^{9}$ Hemano et al. and Nosadini et al. established that increased renal arterial resistance strongly predicts the course and outcome of renal function in type II diabetes with microalbuminuria. So RI remained significant and independent risk factor for the presence of albuminuria. ${ }^{10,11}$

RI value is found to increase with progression of disease and therefore useful for predicting deterioration in renal function. ${ }^{14}$ By studying the blood flow characteristics, disease affecting the vascular and tubointerstitial compartment of the kidneys. This study was designed to throw some light in these aspects.

With the rise in the prevalence of diabetes worldwide there is expected rise in diabetic nephropathy. So, a highly accurate non-invasive and specific diagnostic tool for the detection of subtle renal changes that reflects the presence of diabetic nephropathy is highly desired.

Conventional ultrasonography though useful in evaluating the morphology of the kidneys only qualitatively can assess the renal cortical echogenicity which reflects the degree of histopathological change within the renal parenchyma but provide no information regarding functional abnormality of the kidneys.

Use of Computed Tomography (CT scan) in diagnosis of renal parenchymal disease is very 
limited. It provides indirect evidence of diseases affecting vascular compartment of kidney thus, the parameters for assessing renal function status are indirect and non-specific.

Magnetic Resonance Imaging (MRI) depicts loss of cortical intensity and corticomedullary differentiation in diffuse parenchymal disease. It is unrelated to the type of parenchymal disease and do not allow differentiation of parenchymal diseases. ${ }^{15}$

Amongst the radiological diagnostic modalities employed in diagnosis of diabetic nephropathy, the renal resistive indices measurement by duplex Doppler ultrasonography was found to have more association with the organ-specific changes and allows the direct visualization and assessment of intrarenal arteries where the changes of diabetic nephropathy are found. ${ }^{16}$ Comparison of conventional and duplex Doppler ultrasonography shows duplex Doppler ultrasonography is superior to conventional ultrasonography in the detection of renal abnormalities in clinically silent as well as manifest diabetic nephropathy. ${ }^{6}$ Resistive index values start increasing in diabetic subjects even before appearance of microalbuminuria. ${ }^{17}$

Objective of the study was to evaluate the diagnostic usefulness of renal resistive index by duplex Doppler ultrasonography for detection of renal dysfunction in diabetic patients and also to compare renal RI as a diagnostic tool for diagnosis of diabetic nephropathy with the biochemical diagnosis.

\section{Materials and Methods}

Total number of 53 subjects were enrolled in this present cross sectional study in the department of Radiology and Imaging, Bangabandhu Sheikh Mujib Medical University (BSMMU) in collaboration of Nephrology of the same institution during two years from July 2011 to June 2013 aim to evaluate the diagnostic usefulness of renal resistive index by duplex Doppler ultrasonography for detection of renal dysfunction in diabetic patients. Clinically diagnosed diabetic patients having diabetic nephropathy referred to the department of Radiology and Imaging in BSMMU for ultrasonography of KUB region or whole abdomen were selected as sample. Diabetic nephropathy was diagnosed by the Persistent albuminuria ( $>300 \mathrm{mg} / \mathrm{d}$ or $>200 \mu \mathrm{g} / \mathrm{min}$ ) that is confirmed on at least two occasions 3-6 months apart, Progressive decline in the glomerular filtration rate (GFR) and Elevated arterial blood pressure by the physician. However, Serum creatinine level more than $1.3 \mathrm{mg} / \mathrm{dl}$ was also considered as having diabetic nephropathy. Also patient having albumin in urine more than $300 \mathrm{mg} / \mathrm{dl}$ was considered to have microalbuminuria.

After informing all the necessary information regarding the research study, data were collected in a pre-designed structured data collection sheet. Before performing ultrasonography, proper counseling and reassurance to the patient regarding the procedure was done. Both the kidneys were visualized by commercially available real time scanner (GE Voluson) equipped with a curvilinear transducer operating at 3.5 MHz. Right kidney was visualized with the patient in supine position through the liver. For the left kidney, the patient was asked to be in left sideup position with the left arm extended over the head.

Patient was asked to suspend respiration or perform quiet breathing. First of all, kidneys were visualized with real time B-scan and then intrarenal arteries were examined by duplex Doppler ultrasound (Illustration 1). At least three measurements of intrarenal arteries were taken in upper, mid and lower intrarenal arteries of each kidney. The RI value was determined by preset formula incorporated in the machine. Only those showing the highest RI values obtained with duplex Doppler were taken into account (Illustration 1).

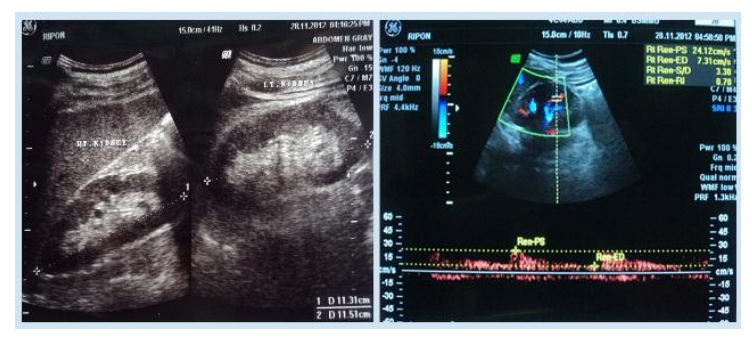

A. Conventional ultra-sonogram of kidneys with parenchymal echogenicity less than liver parenchyma

B. Doppler ultra-sonogram with resistive index (RI) value of 0.70 


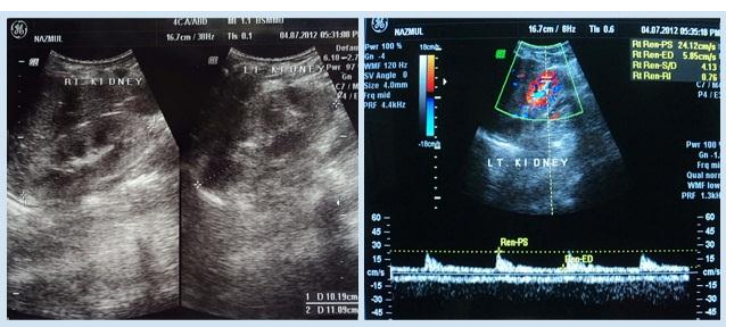

A. Conventional ultra-sonogram of kidneys with parenchymal echogenicity same as liver parenchyma

B. Doppler ultra-sonogram with resistive index (RI) value of 0.76
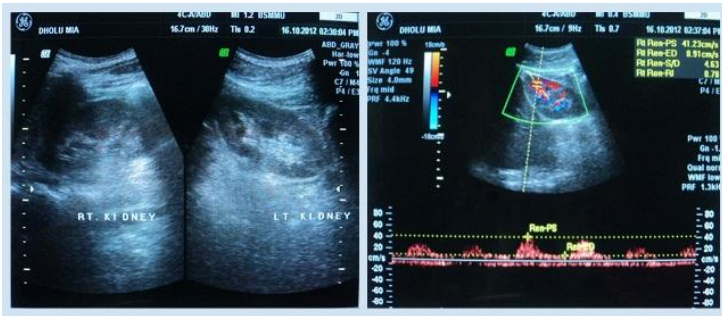

A. Conventional ultra-sonogram of kidneys with parenchymal echogenicity same as liver parenchyma

B. Doppler ultra-sonogram with resistive index (RI) value of 0.78

Statistical analyses of the results were obtained by using window based computer software devised with Statistical Packages for Social Sciences (SPSS-20). The results were presented in tables, figures, diagrams. The test statistics used were descriptive statistics, Pearson's correlation test. Level of significance was set at 0.05 and $\mathrm{p}<0.05$ was considered significant.

\section{Result}

It was observed that majority $(45.3 \%)$ patients belonged to 51-60 years of age. The mean age was found to be $52.66 \pm 7.4$ years (Table I). Gender distribution was that, there were $29(54.7 \%)$ patients were male and female were $24(45.3 \%)$. Male female ratio was 1.2:1 (Fig. 1).

Table I: Distribution of the study patients by age $(\mathrm{n}=53)$

\begin{tabular}{|c|c|c|}
\hline Age (years) & Frequency & Percentage \\
\hline $31-40$ & 6 & 11.3 \\
\hline $41-50$ & 17 & 32.1 \\
\hline $51-60$ & 24 & 45.3 \\
\hline$>60$ & 6 & 11.3 \\
\hline $\begin{array}{c}\text { Mean } \pm \text { SD } \\
\text { Min-max }\end{array}$ & $\begin{array}{r}52.6 \\
(38\end{array}$ & $\begin{array}{l} \pm 7.4 \text { years } \\
55 \text { ) years }\end{array}$ \\
\hline
\end{tabular}

Fig. 1 shows resistive index of study population, RI was $\geq 0.7$ in $39(73.6 \%)$ and $<0.7$ in $14(26.4 \%)$ of study population. Mean resistive index was $0.71 \pm 0.04$ and it ranged from $0.6-0.82$ (Fig. 1).

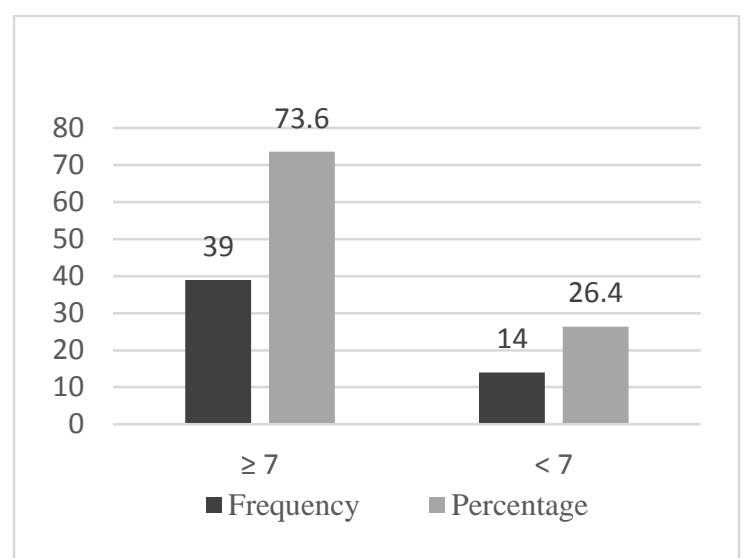

Fig. 1: Bar diagram showing distribution of the study patients by resistive index $(\mathrm{RI})(\mathrm{n}=53)$.

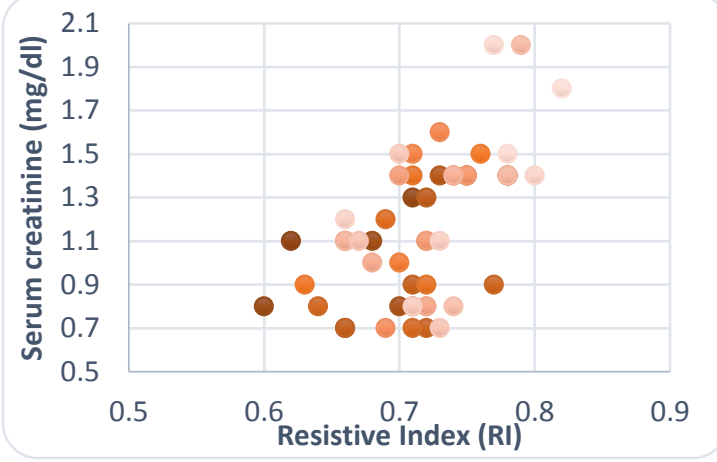

Fig. 2: Scatter diagram showing positive correlation and significant with RI and serum creatinine $(\mathrm{r}=0.581$; $\mathrm{p}<0.01)$.

Positive correction between resistive index with serum creatinine $(\mathrm{r}=0.581, \quad \mathrm{p}<0.01)$ and albuminuria $(\mathrm{r}=0.725, \mathrm{p}<0.01)$ were observed (Fig. 2 and Fig. 3).

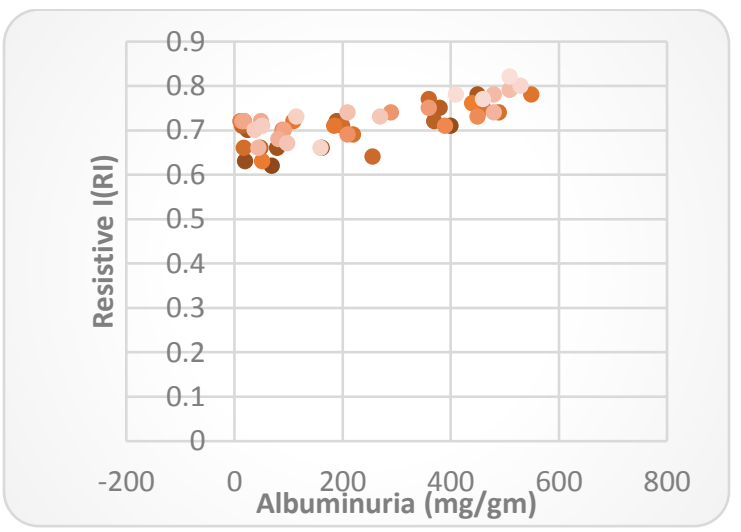

Fig. 3: Scatter diagram showing positive correlation and significant with RI and albuminuria $(r=0.725 ; \mathrm{p}<0.01)$. 
Positive correction between resistive index with serum creatinine $(\mathrm{r}=0.581, \quad \mathrm{p}<0.01)$ and albuminuria $(r=0.725, p<0.01)$ revealed that renal function decreased with increased RI of renal artery.

\section{Discussion}

The duplex Doppler ultrasonography has made advancement in the field of diagnostic imaging with emerging importance as a diagnostic modality. In this current study it was observed that majority of the patients were in sixth decade with the mean age of $52.66 \pm 7.4$ years, ranging from 38 to 65 years. A recent study ${ }^{19}$ have shown in their series, the mean age of patients with diabetes as $57.4 \pm 7.6$ years. In another study ${ }^{20}$ it was showed the mean $( \pm \mathrm{SD})$ age of the patients having diabetes as $55.9 \pm 12.8$ years which is comparable with the current study. Researchers ${ }^{21}$ found the mean age of the patients with diabetes $47.8 \pm 11.34$ years. Similarly, another study ${ }^{22}$ had revealed the mean \pm SD age of patients with having diabetes was $43.6 \pm 3.1$ years $(p=0.019)$. Similar age range was also obtained in another study. ${ }^{23}$

In this present study it was observed that male was predominant in both groups, where male was found as $54.7 \%$. Male to female ratio was 1.2:1 in the study patients. Researchers ${ }^{8}$ found male $69.6 \%$ in patients with diabetes which is closely resembled with the present study. Similarly, male predominance was also observed by researchers. ${ }^{19-22}$ However, a comparable study ${ }^{16}$ found female $57.1 \%$ in patients with diabetes.

It was observed that resistive index $(\geq 0.7)$ was $73.6 \%$ in patients with diabetes. Mean resistive index was found to be $0.71 \pm 0.04$ and varied from 0.60 to 0.82 . Similarly, it ${ }^{19}$ was found RI significantly higher in diabetic patients $(0.69 \pm 0.05)$. In another study 9 it was showed the mean RI value $(0.69 \pm 0.1)$ in patients with diabetes. Similar results about the resistive index were also made by other researchers. ${ }^{21}$ In another study it was seen that mean RI of $0.64+/-0.09$ in 23 patients with early diabetic nephropathy. Patients with established nephropathy had a mean RI of $0.83+/-0.11 .5$

A positive correction $(\mathrm{r}=0.581, \mathrm{p}<0.01)$ was found between resistive index with serum creatinine and albuminuria which indicates deterioration of renal function, i.e. progression of the disease. In previous study ${ }^{9}$ it was shown that the correlation between serum creatinine and RI values in diabetic nephropathy to be $(r=0.84)$. Similarly, study $^{20}$ found correlation between the two to be $\mathrm{R} 2=0.67(\mathrm{p}<0.001)$. A positive correlation and significant $(r=0.725, p<0.01)$ was found between albuminuria and renal resistive index. An article ${ }^{10}$ revealed that there was a significant association between urinary albumin excretion and RI. Researchers ${ }^{24}$ found that resistive index (RI) was highest in the patients with microalbuminuria. Afsar and Elsurer ${ }^{21}$ also observed that renal resistive index was highest in patients with increased 24-h urinary albumin.

Conclusion: It could be concluded that mean resistive index was significantly higher in diabetic patients suspected of developing nephropathy and resistive index correlated well with level of serum creatinine and albumin in urine. So, resistive index measured by Duplex Doppler ultrasonography has value in identifying diabetic patients who are developing nephropathy and can be used as an additional diagnostic tool in diagnosing diabetic nephropathy as well as to monitor progression of nephropathy.

\section{References}

1. Hamed SRSB, Pavkovic P, Metelko Z. Microalbuminuria and diabetes mellitus. Diabetologia Croatica, 2002; 2: 31-34.

2. Ritz E, Orth SR. Nephropathy in patients with type 2 diabetes mellitus. N Engl J Med, 1999; 341(15): 1127-33.

3. Moghazi S, Jones E, Schroepple J, Arya K, McClellan W, Hennigar RA et al. Correlation of renal histopathology with sonographic findings. Kidney Int, 2005; 67(4): 1515-20.

4. Hricak H, Cruz C, Romanski R, Uniewski MH, Levin NW, Madrazo BL. Renal parenchymal disease: sonographic-histologic correlation. Radiology, 1982; 144(1): 141-47.

5. Platt JF, Rubin JM, Ellis JH. Diabetic nephropathy: evaluation with renal duplex Doppler US. RSNA Radiology 1994; 190 (2): 35-40.

6. Soldo D, Brklijacic B, Bozikov V, Drinkovic I, Hauser M. Diabetic nephropathy. Comparison of conventional and duplex Doppler ultra-sonographic findings. Acta Radiol 1997; 38(2):296-302

7. Kim SH, Kim SM, Lee HK, Kim S, Lee JS, Han MC, 1992. Diabetic nephropathy: duplex Doppler ultrasound findings. Diabetes Research and Clinical Practice, 18(2), pp. 75-81 
8. Ohta Y, Fujii K, Arima H, Matsumura K, Tsuchihashi $\mathrm{T}$, Tokumoto $\mathrm{M}$, et al. Increased renal resistive index in atherosclerosis and diabetic nephropathy assessed by Doppler sonography. J Hypertens 2005; 23(10): 1905-11.

9. Sari A, Dinc H, Zibandeh A, Telatar M, Gümele HR. Value of resistive index in patients with clinical diabetic nephropathy. Invest Radiol 1999; 34(11): 718-21.

10. Hamano K, Nitta A, Ohtake T, Kobayashi S. Associations of renal vascular resistance with albuminuria and other macroangiopathy in type 2 diabetic patients. Diabetes care 2008; 31(9): 1853-57.

11. Nosadini, R., Velussi, M., Brocco, E., Abaterusso, C., Carraro, A., Piarulli, F., et al. 2006. Increased renal arterial resistance predicts the course of renal function in type 2 diabetes with microalbuminuria. Diabetes, 55(1), pp.234-39.

12. Milovanceva-Popovska M, Dzikova S. Progression of diabetic nephropathy: value of intrarenal resistive index (RI). Prilozi, 2007; 28(1): 69-79.

13. Ljubic S, Brkljacic B, Metelko Z, Pavlic-Renar I. Renal resistance index in type 2 diabetes. Scientific Paper (2006).

14. Ikee R, Kobayashi S, Hemmi N, Imakiire T, Kikuchi $\mathrm{Y}$, Moriya $\mathrm{H}$, et al. Correlation between the resistive index by Doppler ultrasound and kidney function and histology. Am J Kidney Dis 2005 46(4): 603-09.

15. Kelsey FI, Webb JAW, 2001. Renal parenchymal disease. In: Grainger and Allison's Diagnostic Radiology, A textbook of Medical Imaging. Ed. by Grainger R.G, Allison D.J, Adam A.D.A.K. (Editors). Churchill Livingston, London. pp.1541-42.

16. Ishimura E, Nishizawa Y, Kawagishi T, Okuno YS, Kogawa K, Fukumot S. Intrarenal hemodynamic abnormalities in diabetic nephropathy measured by duplex Doppler sonography. Kidney International 1997; 51: 1920-27.
17. Thukral A, Mishra M, Srivastav V, Kumar H, 2011. Determinants of intravascular resistance in indian diabetic nephropathy patients: a hospital-based study. International Journal of Vascular Medicine, 2011

18. Zwain AAM, Khudhair SA. Intrarenal hemodynamic changes in type 2 diabetic patients. Kufa Med.Journal, 2010; 13(1): 23-37.

19. Masulli M, Mancini M, Liuzzi R, Daniele S, Mainenti PP, Vergara $E$ et al. prediction of diabetic nephropathy. Nutr Dis 2009; 19(5): 358-364.

20. Nejad MN, Jafari B, Alipour P. Arterial Resistive Index (RI) in Type II Diabetic Nephropathy Stages and Healthy Controls. Iran J Radiol 2009; 6(1): 2931.

21. Afsar B, Elsurer R. Short Report: Complications Comparison of renal resistive index among patients with Type 2 diabetes with different levels of creatinine clearance and urinary albumin excretion. Diabet. Med. 2012; 29: 1043-46.

22. Ghafoori M, Shiva SH. The Relation between Serum Creatinine and Renal Resistance Index in Parenchymal Kidney Disease. Iran. J. Radiol 2007; 4(2): 109-11.

23. Sperandeo M, D'Amico G, Varriale A, Sperandeo G, Annese MA, Correra M. Pulsed-wave color Doppler echography of the intrarenal vessels in patients with insulin-dependent diabetes mellitus and incipient nephropathy. Arch Ital Urol Androl 1996; 68: 18387.

24. Fallah M, Nafisi-Moghadam R, Nouri N. Relationship between Intra-renal Arterial Resistance Index (RI) and Albuminuria in Diabetic Patients. Iranian Journal of Diabetes and Obesity 2012; 4: 710 